Revue des patrimoines

$31 \mid 2017$

Patrimoines de la santé : essais de définition - enjeux de conservation

\title{
L'inventaire du patrimoine culturel immatériel hospitalier. Une histoire de résilience(s)
}

An inventory of the intangible cultural heritage of a hospital, a story of resilience

\section{Yann Leborgne}

\section{OpenEdition}

\section{Journals}

Édition électronique

URL : http://journals.openedition.org/insitu/14134

DOI : $10.4000 /$ insitu. 14134

ISSN : 1630-7305

\section{Éditeur}

Ministère de la Culture

\section{Référence électronique}

Yann Leborgne, «L'inventaire du patrimoine culturel immatériel hospitalier. Une histoire de résilience(s) », In Situ [En ligne], 31 | 2017, mis en ligne le 22 février 2017, consulté le 09 octobre 2020. URL : http://journals.openedition.org/insitu/14134 ; DOI : https://doi.org/10.4000/insitu.14134

Ce document a été généré automatiquement le 9 octobre 2020.

\section{(i) $\odot$

In Situ Revues des patrimoines est mis à disposition selon les termes de la licence Creative Commons Attribution - Pas d'Utilisation Commerciale - Pas de Modification 4.0 International. 


\title{
L'inventaire du patrimoine culturel immatériel hospitalier. Une histoire de résilience(s)
}

\author{
An inventory of the intangible cultural heritage of a hospital, a story of \\ resilience
}

Yann Leborgne

1 Au fil des projets qui en dressent progressivement l'inventaire, le patrimoine culturel immatériel dévoile l'extraordinaire diversité de ses manifestations et lieux d'expression, ce dont témoigne la définition de l'Unesco. Selon la convention adoptée en 2003, le patrimoine culturel immatériel regroupe les "pratiques, représentations, expressions, connaissances et savoir-faire - ainsi que les instruments [...] et espaces culturels qui leur sont associés - que les communautés, les groupes et [...] les individus reconnaissent comme faisant partie de leur patrimoine culturel». Transmis de génération en génération, il "est recréé en permanence par les communautés et groupes en fonction de leur milieu, de leur interaction avec la nature et de leur histoire ». Il « leur procure un sentiment d'identité et de continuité, [et] contribu[e] à promouvoir le respect de la diversité culturelle et la créativité humaine. $»^{1}$

Outre le fait que cette définition signale à quel point le patrimoine culturel immatériel recouvre un ensemble très vaste de phénomènes sociaux, il importe de souligner combien elle insiste aussi sur leur caractère dynamique. C'est-à-dire que, tandis que l'immatérialité patrimoniale se perpétue, ses manifestations présentent la particularité d'être évolutives. Elles se transforment tout en s'adaptant aux époques traversées. Cette malléabilité renvoie directement aux enjeux qui président à la transmission du patrimoine culturel immatériel. Elle n'a pas alors seulement trait au fait qu'un patrimoine culturel immatériel qui se fige deviendrait vulnérable aux aléas de l'histoire. Elle a d'abord à voir avec la capacité des groupes humains à assurer leur continuité par-delà les usures du temps, les chocs ou les traumatismes qui les perturbent au fil de leur existence. 
Ces considérations nous conduisent à esquisser l'hypothèse d'une relation entre l'expression du patrimoine culturel immatériel et des phénomènes de résilience ${ }^{2}$.

4 Afin d'avancer dans cette réflexion, nous revenons dans cet article sur un projet d'inventaire initié au CHU de Rouen en 2013-2014. Celui-ci met en lumière la façon dont l'exploration de l'immatérialité patrimoniale peut participer du soin d'une communauté hospitalière et comment la démarche accompagne une re-connaissance identitaire.

\section{Du patrimoine immatériel dans les territoires rationnels du soin}

5 Au regard des manifestations couramment concernées par des opérations d'inventaire du patrimoine culturel immatériel, il peut sembler surprenant que des établissements hospitaliers universitaires se livrent à pareille entreprise. Ces territoires sont en effet habités par la rationalité scientifique, les pratiques médicales et paramédicales les plus en pointe, sans oublier le réalisme très cadré de leur administration.

Pourtant, au-delà de ces considérations techniques, il faut aussi se rappeler que les hôpitaux sont des communautés d'hommes et de femmes qui œuvrent à l'accueil des souffrants. Ils accompagnent des êtres à des étapes fondamentales de leur existence, qu'il s'agisse de la traversée d'une maladie jusqu'à sa guérison, de la naissance ou lors de la mort. Ainsi, la prise en charge hospitalière sécrète une puissante chaîne signifiante où l'humanité, dans ce qu'elle a de plus vulnérable, questionne ses limites.

7 Les hôpitaux éprouvent d'ailleurs eux-mêmes sans cesse leurs limites. D'abord parce qu'ils s'inscrivent dans des territorialités régionales, nationales voire internationales. Les centres hospitalo-universitaires s'insèrent dans les espaces urbains de villes capitales (fig. 1). Ils s'avèrent étroitement liés à l'expression du pouvoir sur un territoire. Mais surtout, ces établissements sont d'abord organisés pour répondre à la demande de soins de la société qui leur confie ce rôle. Ils doivent s'adapter à ses évolutions et mutations. L'identité hospitalière se renouvelle ainsi au rythme des transformations de son environnement global.

Figure 1

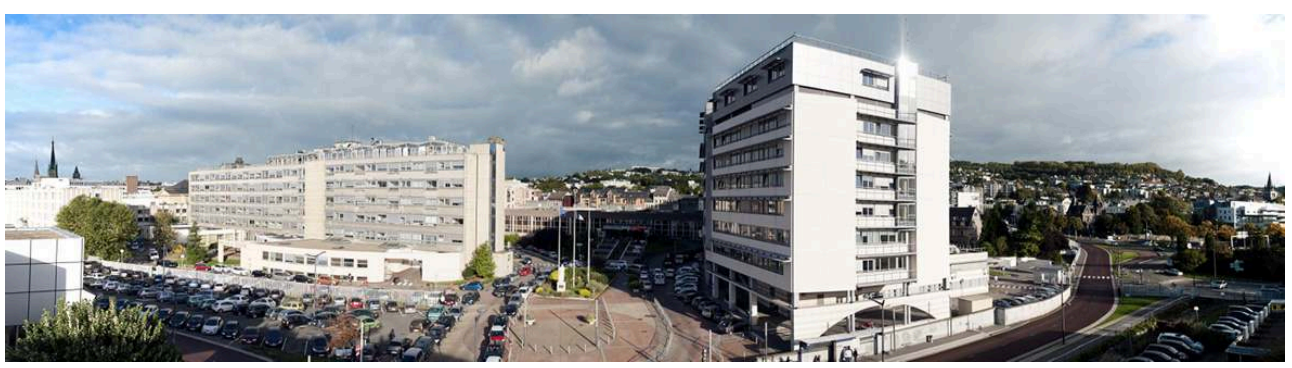

L'entrée principale du CHU de Rouen - Charles Nicolle, 2013.

Phot. CHU-Hôpitaux de Rouen. (c) CHU de Rouen. 


\section{Une démarche pour soigner une communauté en quête de sens}

8 La période actuelle constitue précisément l'un de ces moments où se produisent d'importantes transformations. Dans un contexte financier très contraignant, la réorganisation des hôpitaux publics en «pôles" et la disparition des anciens « services » accompagnent une mutation économique (traduite par la loi instaurant la tarification à l'acte $)^{3}$ qui conduit ces établissements à adopter un modèle de fonctionnement plus libéral. Ce mouvement se conjugue avec un changement des relations qu'entretiennent les patients et les soignants. Tandis que les «usagers " se perçoivent de plus en plus souvent en tant que «clients » des hôpitaux, du côté des soignants l'image du «professionnel exerçant un métier » tend à supplanter celle de l'agent public. Ainsi, une certaine individualisation des rapports sociaux semblerait progresser en ces lieux dédiés aux soins de tous.

9 Ces évolutions paraissent éveiller de sérieux troubles chez les hospitaliers, qu'ils soient médicaux, paramédicaux, administratifs ou techniques. Les agents déplorent en effet un émiettement des relations socioprofessionnelles ainsi qu'une dévalorisation du travail ${ }^{4}$. Ils craignent également la disparition de l'identité publique de leurs établissements. En somme, ils expriment une souffrance véritable; celle dont témoigne notamment la fréquence de l'absentéisme et des grèves. C'est cette souffrance qui a interpellé la délégation culturelle du CHU de Rouen et c'est pour y répondre qu'elle énonça en 2012, auprès de la DRAC, le souhait d'interroger et de mettre en valeur l'identité des métiers hospitaliers.

10 Présentée comme un projet à fois culturel et social, la démarche de cette délégation fut d'emblée orientée vers l'intérieur comme vers l'extérieur du CHU.

11 Vers l'intérieur, il s'agissait d'interroger ce qui unifie en ces lieux un ensemble humain aussi vaste et diversifié. Autrement dit, il semblait qu'une réponse à la dévalorisation et au manque de reconnaissance des hospitaliers ne pouvait faire l'économie d'un processus introspectif. Il viserait une re-connaissance, au sens de la production d'un nouveau savoir du collectif sur ce qu'il est et sur les valeurs qu'il porte.

12 Ce questionnement ne devait pas induire un repli dans l'« entre-soi ». C'est la raison pour laquelle la délégation culturelle désira l'intervention d'un chercheur venu de l'extérieur. L'hôpital se définissant à travers son rôle vis-à-vis de la société à laquelle il appartient, l'opération devait proposer une nouvelle représentation des pratiques qui s'y jouent en dévoilant au public les significations méconnues que sécrète cet univers de soins. L'enjeu culturel et social rejoignait alors une problématique communicationnelle. C'est à ce titre que le projet fut soutenu par la direction de la communication du CHU et la Ville de Rouen.

13 Il semble important de souligner ici que cette quête identitaire autour des métiers était initialement formulée par la délégation culturelle du CHU sous l'angle d'une « collecte de la mémoire vivante » mais des entretiens préalables engagés auprès de ces délégués ont révélé que cette formulation ne correspondait pas tout à fait à leur désir. En effet, ceux-ci ne souhaitaient pas mettre en lumière le récit d'un passé « historique » porteur de message, d'un enseignement des « anciens » adressé pour le présent et l'avenir de la communauté. Ils appelaient "mémoire» une substance immatérielle, reçue et transmise, qui « donne vie» aux pratiques et savoir-faire hospitaliers contemporains. 
Leur demande se portait donc sur l'exploration du présent. En interrogeant les pratiques actuelles, il était avant tout question d'aller à la recherche des registres symboliques et imaginaires intemporels, intangibles, inconsciemment hérités, qui donnent sens au travail des hospitaliers. Se plaçant au-delà du «faire» et de la matérialité des actes soignants, sur les conseils de la DRAC, ils choisirent d'orienter leur démarche vers un inventaire du patrimoine culturel immatériel ${ }^{5}$.

\section{Reconstruire une chaîne signifiante}

Un inventaire du patrimoine culturel immatériel hospitalier représente un défi dans la mesure où il ne peut reposer sur une description de pratiques, tant celles-ci se révèlent d'une évolutivité extrême. Cette recherche s'est donc fondée sur l'hypothèse selon laquelle ces pratiques hospitalières tendraient à se transformer tout en sauvegardant une chaîne signifiante constituant leur immatérialité patrimoniale. Il s'agit par exemple ici de ce que d'aucuns appellent l'humanité du soin, dont il est toujours craint qu'elle soit transgressée, comme si un hôpital luttait sans cesse contre le risque de sa déshumanisation. Sur cette base, ce travail devait contribuer à mettre en lumière (ou à « inventorier ») un ordre symbolique et imaginaire, lié à la "sauvegarde de l'humanité du soin ", qui épouserait au sein d'un établissement hospitalier la rationalité des exercices professionnels. Chaque professionnel, chaque métier, serait en principe détenteur d'une partie de cet ordre à reconnaître.

15 L'entreprise est bien sûr considérable si l'on mesure le gigantisme et la complexité d'un hôpital universitaire. Le seul $\mathrm{CHU}$ de Rouen regroupe par exemple près de 10000 agents qui se répartissent dans plus de 140 métiers répertoriés. Dès lors, l'exploration de son patrimoine culturel immatériel ne peut s'effectuer que sur plusieurs années ; un peu comme la lente reconstitution des séquences d'un " génome " qui ne serait pas biologique mais culturel, celui d'une communauté hospitalière faisant corps avec son territoire.

\section{L'hôpital, un territoire de la délivrance}

16 L'approche territoriale ${ }^{6}$ fut choisie comme pierre angulaire du déroulement de cette recherche. Ce choix a découlé en partie de la discipline du chercheur ayant en charge cet inventaire, la géographie induisant ce regard spatial sur les phénomènes humains. Cependant, nous avons également suivi une piste suggérée par l'ancien doyen de la faculté de médecine de Rouen, le professeur Lauret, pour qui « un hôpital, c'est d'abord un territoire $»^{7}$. Un territoire qui englobe d'autres territoires, les corps souffrants des patients...

17 Par ailleurs, il est vrai que la tradition européenne exprime un rapport étroit entre le soin et le territoire. Évoquons à cet égard les rois thaumaturges ${ }^{8}$, ces souverains d'Ancien Régime auxquels on prêtait le pouvoir de guérir les écrouelles par le toucher. Ces croyances plaçaient le roi dans un rôle privilégié d'intercesseur auprès de la puissance céleste, d'ordre divin (Dieu, d'où il légitimait son autorité). Elles traduisaient un transfert de territorialité des sujets vers leur souverain. En lui confiant rituellement leur corps, ses sujets reconnaissaient au roi son pouvoir d'opérer, de pénétrer leur territoire pour les libérer de la souffrance. 

Citons par exemple l'image des " médecins-rois ${ }^{9}$ " assez connue en milieu hospitalier. Mais au-delà, il nous faut également souligner que, dès lors qu'ils se confient à une communauté hospitalière, les patients ne s'appartiennent plus tout à fait. Ils sont dépossédés d'une part de leur autorité naturelle sur leur corps-territoire, car c'est aussi de la dépossession induite par la prise en charge que doit survenir la délivrance que constitue une guérison.

\section{Sauvegarder l'humanité par le soin et l'accueil de tous}

L'idée selon laquelle les hôpitaux seraient des territoires de délivrance, incorporant en leur sein des patients pour les libérer de ce qui les étreint, n'est pas une spécificité des établissements publics. Elle est autant valable pour le secteur privé. Cependant, «l'accueil de tous » est une dimension clairement revendiquée et constitutive de leur identité. Elle implique que ces hôpitaux réinscrivent les patients dans une territorialité globale et qu'ils incluent tous les hommes dans un même ensemble. On reconnaît là un caractère religieux et l'on entrevoit un héritage de l'époque où les hôpitaux relevaient de la charité chrétienne (fig. 2). Ils manifestaient un soin accordé à tous, jusqu'aux plus pauvres. Mais c'est également à travers ce prisme que l'on mesure encore l'importance considérable que ces établissements accordent au respect de ce principe, surtout à l'heure où le flux des malades et les contraintes financières pourraient les conduire à y renoncer.

\section{Figure 2}

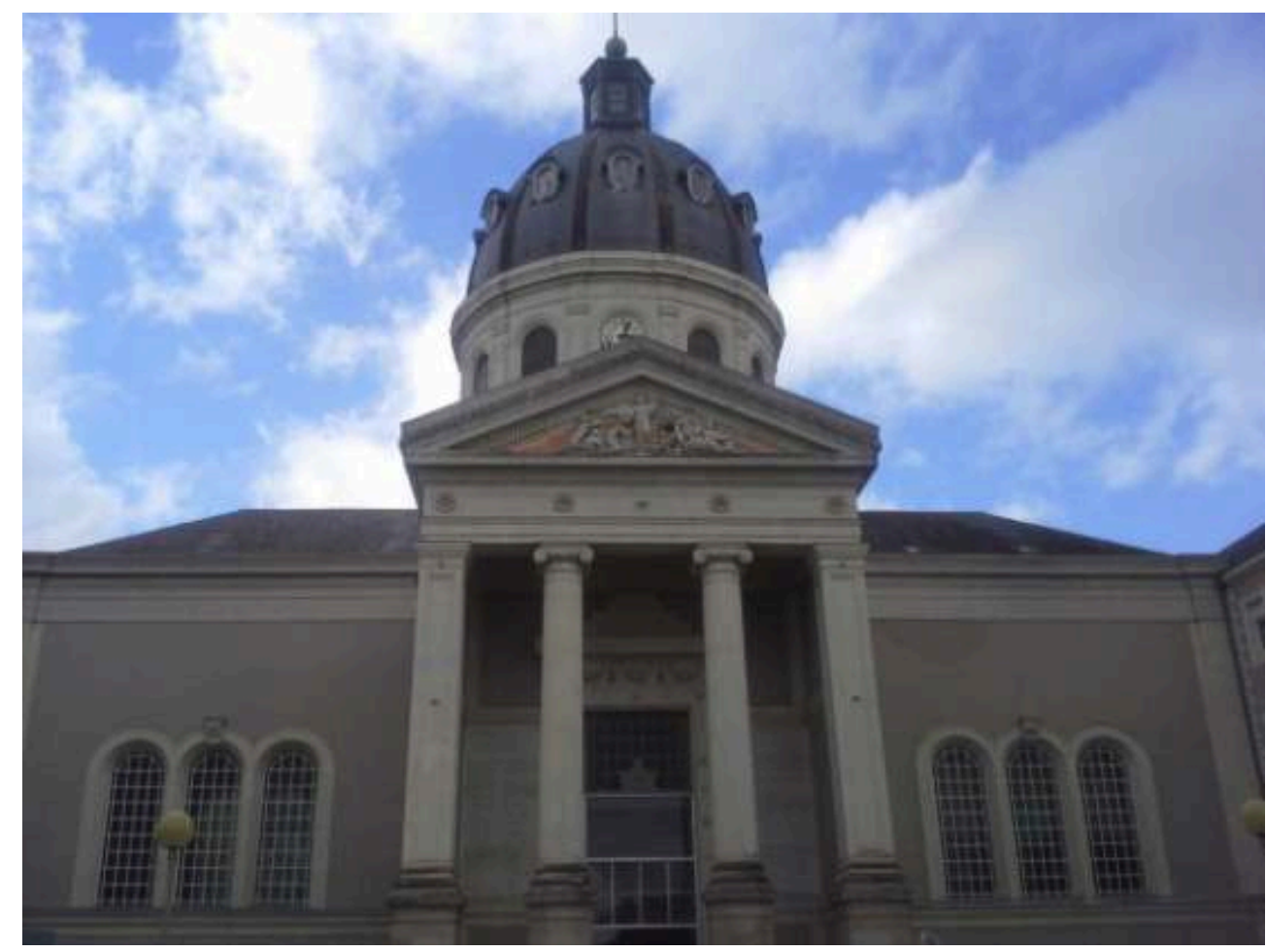

La chapelle du CHU d'Angers où se fait de nos jours l'accueil des patients.

Phot. Leborgne, Yann, 2014. EPCC La Fabrique de patrimoines en Normandie. (c) CRéCET de BasseNormandie. 
jalons posés, on observe qu'un hôpital n'est pas seulement le lieu d'une guerre contre la maladie, dont l'unique finalité serait la guérison organique. Il est également celui d'une lutte pour sauvegarder un ordre fragile où la prise en charge de l'autre revient à lui reconnaître son appartenance à la communauté des hommes, son humanité.

21 Soulignons que cette reconnaissance ne réside pas uniquement dans l'expression d'une appartenance à une communauté globale. S'il est clair qu'en étant pris en charge à l'hôpital le patient se voit resitué dans un grand ensemble, un autre enjeu réside en effet aussi dans la préservation de sa singularité. La signature de communication du CHU de Rouen illustre ce propos: "L'excellence au service de chacun " répond aux attentes d'une société libérale où les malades aspirent à une personnalisation de leurs soins mais ce "chacun" constitue aussi un rappel pour des hospitaliers enclins à effacer les individualités au profit d'une globalité. Ainsi, au sein d'un territoire hospitalo-universitaire qui universalise, tandis que le patient pourrait y devenir un objet indifférencié, l'institution formule la promesse d'une « excellence » qui, en s'adressant à tous, ambitionne de respecter l'être ${ }^{11}$.

Par cette ambition du respect de la singularité, on perçoit combien les pratiques hospitalières sont liées à un bornage entre ce qui est intérieur et extérieur au corpsterritoire. Il s'agit d'abord du territoire d'un être souffrant qui, dès qu'il devient un patient, transfère vers la communauté soignante une part de l'autorité qu'il exerce sur son espace le plus intime, son propre corps. C'est par ce transfert de territorialité que les soignants reçoivent le pouvoir d'intervenir de façon plus ou moins intrusive sur ce patient. Et c'est à partir de là que la limite de son corps-territoire peut être traversée sans être transgressée, une pénétration majeure qu'autorise par exemple l'anesthésie au cours d'une opération chirurgicale. Cette traversée consentie, physique (au cours d'une opération ou d'un soin intrusif), ou psychique (via l'empathie, notamment) fait indéniablement partie de l'identité hospitalière dès lors que cet acte touchant au corps et à son intériorité vise la libération de l'être souffrant.

\section{Sur le terrain d'opération}

Ce travail d'inventaire du patrimoine culturel immatériel fut engagé en reposant sur les acquis précieux des recherches en ethnologie hospitalière réalisées par Marie-Christine Pouchelle. L'approche du terrain hospitalier s'est néanmoins centrée sur une problématique patrimoniale, prolongeant la démarche mémorielle développée par Anne Monjaret sur des hôpitaux parisiens, et elle s'est axée sur un corpus théorique autour de la territorialité issu de la géographie sociale. Une force de l'inventaire du patrimoine culturel immatériel réside dans sa capacité à mobiliser des disciplines en fonction des sujets à investir.

Sur le plan opérationnel, cette recherche s'est fondée sur deux sessions d'enquêtes qui se sont déroulées au CHU de Rouen (printemps 2013) et au CHU d'Angers (printemps 2014). Ces enquêtes ont consisté en un important recueil d'entretiens semidirectifs conduits auprès de professionnels qui avaient été préalablement invités, via un appel à témoignages très largement diffusé ${ }^{12}$, à parler du métier qu'ils exercent à l'hôpital. Les propos de ces volontaires ont permis de révéler un certain nombre de phénomènes qui furent formalisés dans une «fiche d'inventaire ${ }^{13}$ » dans la mesure où 
ils mettaient en lumière un système symbolique et imaginaire autour duquel se structure la pratique de leur métier.

Compte tenu du volume important d'informations déjà délivré par ces témoins (111 heures d'entretiens collectées grâce à la participation d'une centaine d'agents sur un peu plus de 30 services hospitaliers à Rouen et Angers), la réalisation de cette fiche d'inventaire est toujours en cours et elle s'étendra très vraisemblablement sur plusieurs années. Elle s'organise en séquences qui, à terme, devraient finir par s'articuler les unes aux autres pour reconstituer l'architecture immatérielle du patrimoine culturel hospitalier.

Sept séquences (ou "modules» de la fiche d'inventaire) sont actuellement achevées. Les sujets couverts ont trait aux urgences et au SMUR, au service de cardiologie, au toucher de l'aide-soignante en réanimation, à l'hygiène hospitalière ainsi qu'au travail des préparateurs de la chambre mortuaire. Bien entendu, il est impossible dans le cadre de cet article d'exposer la totalité des résultats de ces premières investigations. Néanmoins, il semble intéressant d'exposer ici un « module » illustrant la façon dont les principes énoncés plus haut permettent de forger une grille d'identification du patrimoine immatériel.

À cet égard, l'étude de la préparation des corps dans la chambre mortuaire constitue un cas parlant. Non qu'il faille présenter un versant sinistre du territoire hospitalier. La chambre mortuaire révèle d'abord combien l'hôpital est aussi un lieu où se perpétuent des rituels marquant les bornes du vivant, où sont questionnées la puissance et l'impuissance soignante, mais aussi la matérialité et l'immatérialité de l'existence humaine.

\section{Une résilience de la vie dans la chambre mortuaire}

Dans un univers hospitalier tout entier tourné vers la sauvegarde de la vie, le service de la chambre mortuaire occupe une place singulière. Il est en effet le lieu par lequel transitent les patients qui n'ont pu être sauvés, ceux pour qui, malgré leur lutte et la qualité de la médecine prodiguée, le combat s'est achevé par le décès. Après que la mort est survenue dans le service de soins, après qu'une dernière toilette y a été dispensée, le corps du défunt est transféré dans cette autre chambre afin d'y être préparé puis présenté à la famille. Ce passage marque sa sortie du territoire hospitalier avant l'inhumation.

29 Au premier abord, la seule mention de la chambre mortuaire évoque une perspective funeste, en totale contradiction avec les attentes que nourrit une prise en charge hospitalière. Pourtant, elle ne saurait être confondue avec le lieu clos de la mort. À l'instar de ce qui se joue dans les autres parties de l'hôpital, l'analyse des pratiques qui s'y jouent révèle que celles-ci sont essentiellement dirigées vers une sauvegarde de la vie humaine.

30 Au-delà de l'apparent paradoxe, cet aspect explique le placement de ce service au CHU de Rouen sous l'égide de la direction des soins. Le témoignage de son responsable (extrait $\mathrm{n}^{\circ}$ 1) est à cet égard éloquent. À travers la notion de "patient décédé », il s'agit d'affirmer une continuité d'appartenance de la chambre mortuaire au territoire hospitalier. Le défunt est ainsi toujours considéré comme un souffrant que l'on soigne. 


\section{Extrait de témoignage $\mathrm{n}^{\circ} 1$}

« Moi je parle de « patient décédé ». Notre philosophie, c'est que l'on est dans un dernier soin au patient qu'on a pris en charge et que, malheureusement, on n'a pas pu guérir. Je suis soignante, je suis infirmière. [...] Je suis dans le soin, je suis cadre de santé. Je suis infirmière et puéricultrice, donc je suis dans le soin. La philosophie que nous avons ici, c'est donc un soin qu'on offre au patient et à sa famille pour lui laisser faire son deuil de façon sereine. Dans les services [...], ils ont vu le patient souffrir, avec le visage crispé par la douleur, la peur, la souffrance. Ici, ils doivent avoir une image apaisée de leur membre de famille. Pour moi, c'est le dernier soin que l'hôpital offre : le patient apaisé. »

Cadre de santé, responsable de la chambre mortuaire, CHU de Rouen

D'après les témoignages, le premier soin délivré dans la chambre mortuaire viserait à « apaiser » le patient décédé dont le corps (on n'emploie jamais le mot « cadavre ${ }^{14}$ ») est souvent marqué par la souffrance tandis qu'il porte les crispations des ultimes douleurs ou les stigmates d'une longue lutte contre la maladie. C'est la raison pour laquelle les préparateurs œuvrant dans le service s'occupent immédiatement (si cela n'a pas déjà été fait dans le secteur de soins) de refermer sa bouche et ses yeux. En effet, non seulement l'apparence du sommeil doit désormais supplanter la fixité de la mort, l'apaisement doit mettre un terme aux tourments auxquels ce patient était jusqu'ici la proie.

Ce qu'expriment à ce sujet les agents est intéressant car, en parlant de soin donné à un être décédé, ils introduisent incidemment l'idée selon laquelle leur pratique libèrerait de ses douleurs une instance immatérielle qu'on pourrait désigner comme étant «l'âme» de ce patient. Pour autant, il leur importe également d'adoucir le choc émotionnel que provoque au sein d'une famille la disparition d'un proche. Le soin exercé sur le corps du défunt est alors aussi dirigé vers cette communauté familiale à laquelle il sera présenté.

Ces premières remarques indiquent que les préparateurs de la chambre mortuaire ne travaillent pas sur de la matière morte. Ils œuvrent avant tout sur ce qui continue de vivre, même s'il s'agit d'une forme d'existence différente de la précédente.

S'agissant du défunt, son existence est désormais idéelle. Elle se présente sous la forme de souvenirs dans la mémoire de ses proches. Les actes réalisés sur ce patient par les agents hospitaliers vont alors viser à lui assurer le meilleur transit de la vie incarnée vers une vie désincarnée. Le souci qu'ils accordent au réaménagement de sa superficialité corporelle relève d'un rituel de passage manifestant un processus spirituel. Il s'agit de médiatiser une traversée s'opérant de l'intériorité vers l'extériorité de son corps-territoire.

Notons que cette traversée se traduit par une étonnante restauration du patient décédé. En effaçant les marques de la maladie et de la mort sur le corps, en lui procurant l'apaisement du sommeil, puis en le maquillant avec une certaine adresse, les préparateurs s'emploient à lui donner une « bonne mine ${ }^{15}$ » comme s'il s'agissait d'une guérison. 


\section{Extrait de témoignage $n^{\circ} 2$}

« Le maquillage on peut le faire à la demande de la famille. Parce que nous, on ne connaît pas les gens. On est plus coutumier du fait qu'une femme se maquille, plutôt qu'une petite grand-mère. Mais des fois, il y a des grand-mères qui se maquillent jusqu'à l'âge de 90 ans avec un petit peu de rouge à lèvres... Donc, la famille nous le demande. [...] On communique [avec elle]. On leur demande de nous ramener une photo et s'ils peuvent [aussi apporter] le maquillage avec lequel elle se maquillait. Pour qu'on soit au maximum fidèle aux couleurs et à l'harmonie de la personne. $[. .$.

[Pour ça] j'ai une expression : [c'est] un maquillage « bonne mine ", avec un peu de fard à joue pour faire croire qu'elle a un peu picolé ! [Rire] Enfin voilà, il faut harmoniser. Il ne faut pas travailler [...] en prenant un pot de peinture. Il faut travailler avec du bon sens. Vous n'allez pas maquiller un homme à chaque fois. [...]. Il vaut mieux ne pas mettre assez de maquillage et en rajouter plutôt qu'en mettre trop. [...] [Il vaut mieux dire] : « ramenez-moi la photo de la dernière cérémonie [...]. [Parce que] la dernière fois qu'il était en cérémonie, ils se sont amusés et ils l'ont vu sourire. »

Arnaud Lejeune, agent de la chambre mortuaire, CHU de Rouen 
possible avec le mythe du mort-vivant). La scène monstrueuse qui en découlerait constituerait un échec pour les préparateurs dont l'effort consiste à sauvegarder l'humanité du défunt décédé.

\section{Extrait de témoignage $n^{\circ} 3$}

« [Quand le corps arrive] il est enveloppé dans des draps. On enlève le drap du dessus. En fonction du service d'où il vient, on a déjà une petite idée [de ce qu'il faudra faire]. [On regarde alors] s'il a eu des perfusions et des pansements, et on les rend étanches. Parce que, derrière ça, on l'habille ; et une fois que la personne est habillée avec ses vêtements définitifs, il ne faut surtout pas que ça coule...

[Bien sûr], il y a déjà une préparation qui a été faite dans le service [de soins], mais entre ce service et nous il y a un transfert qui est fait en véhicule. Les corps sont donc secoués, et si c'est mal méché, ça coule. [Alors, nous] on refait tout cela, car il est hors de question de présenter un corps à la famille dans des draps souillés. [...] Il ne faut pas qu'il y ait d'odeur [...], un risque d'écoulement du nez ou de la bouche...»

Arnaud Lejeune, agent de la chambre mortuaire, CHU de Rouen

Cette sauvegarde d'une identité humaine se révèle être un impératif absolu, pratiquement "sacré », des agents de la chambre mortuaire. C'est en ce sens qu'un corps figé et totalement recroquevillé ne sera jamais exposé tel quel dans le cercueil. Ils veilleront à le redresser en rusant avec des draps ou en utilisant des techniques dont ils détiennent le secret. Et de toute manière, ces opérations devront toujours s'effectuer sans porter atteinte à l'intégrité du défunt car, insistent-ils : «on ne casse jamais un membre».

Ce redressement physique auquel s'emploient ces hospitaliers n'a pas la finalité esthétique d'un " paraître ». Il relève avant tout en effet d'un redressement symbolique de l'être décédé. Cette pratique s'exerce par opposition à une dégradation, au sens d'un état qui marquerait l'emprise de la maladie, de la douleur puis de la mort, une emprise qui tendrait à faire du défunt un être définitivement dé-gradé, déshumanisé.

\section{Extrait de témoignage $n^{\circ} 4$}

« Il y a des pathologies où une personne âgée est recroquevillée... Si elle est pardonnez-moi l'expression - « sur le flanc », je ne vais pas la présenter sur le flanc ! Je vais mettre des draps en dessous pour la rehausser, la remettre droite, pour que les gens l'aient de face. Mais après, ça se discute avec la famille : si la famille a toujours connu cette petite grand-mère là dans le lit, impotente et sur le flanc, je vais d'abord leur poser la question [avant éventuellement de la redresser]. ».

Arnaud Lejeune, agent de la chambre mortuaire, CHU de Rouen

C'est probablement cette lutte où l'on s'efforce de sauvegarder l'appartenance des défunts à la communauté humaine qui explique l'attention particulière que déploient 
les agents de la chambre mortuaire à l'endroit des indigents. La famille ne saurait effectivement se restreindre ici à la parentalité directe devant laquelle un corps est habituellement présenté. Sur le plan signifiant, elle désigne la grande famille des hommes qui ne saurait être hantée par un abandon de ses défunts car elle en serait dégradée de son humanité.

\section{Extrait de témoignage $n^{\circ} 5$}

« [Un défunt] c'est comme le nôtre. C'est comme si c'était l'un des miens.

Que ce soit un enfant ou une personne âgée. Je ne sais pas d'où ils viennent, je ne sais pas leur histoire, mais je les ai en charge. Et ma mission, c'est de les préparer.

Je n'ai pas à savoir ce qu'il a fait ; s'il a été criminel, pédophile ; s'il était alcoolique, SDF...[...] On reste toujours dans un esprit de neutralité. Le jour où je ne suis plus neutre ici, ça ne sert à rien que je continue mon boulot. Le jour où [arrivera un] détenu de la maison d'arrêt de Rouen, pédophile, et que je me dirai « bah tant pis... ", il faudra que je change de métier.

Un indigent ici, il ne repartira jamais nu. Il est enveloppé dans des draps, il a une casaque de l'hôpital, mais il ne me viendrait jamais à l'esprit de le laisser partir nu. »

Arnaud Lejeune, agent de la chambre mortuaire, CHU de Rouen

À travers ces propos, on perçoit le souci de l'accueil de tous ; et finalement, on reconnaît incidemment la figure ancienne de l'ecclesia qui «abrite l'assemblée des hommes ». Il s'agit là de valeurs hospitalières qui s'expriment avec force.

Ainsi, tandis que le cœur du territoire hospitalier est tourné vers la sauvegarde de la vie, les pratiques à l'œuvre dans sa chambre mortuaire ne se distinguent guère de ce combat. Les paroles des agents nous rappellent que, même dans la mort, le défunt reste un patient inscrit dans une même filiation à la fois propre et globale. C'est à ce titre qu'il est soigné et que ce soin traduit le devoir de le garder au sein de la communauté.

Ces dernières indications ne sont pas sans rappeler certaines références villageoises d'autrefois; lorsque les cimetières alors érigés autour des églises (accueillant les vivants) maintenaient aussi les morts à l'intérieur de l'ensemble religieux de la communauté. (Le mot « religieux » est entendu ici au sens du latin «religare», « ce qui relie ».).

Dans ce même ordre d'idées, en œuvrant aux marges du territoire hospitalier, les agents de la chambre mortuaire du CHU de Rouen mettent en œuvre une transition vers l'ailleurs qui ne se réduit pas à des techniques de conservation à seule fin d'exposer un corps. La préparation du patient décédé se révèle être aussi la ritualisation d'un passage vers une forme d'existence libérée de la souffrance. Il s'agit d'un départ vers une vie désincarnée, idéelle et mémorielle, que l'on veut apaisée au regard de la communauté des hommes, mais aussi à distance respectable des vivants. C'est ainsi, en étant demeuré humain, que le corps sans vie de l'être disparu pourra quitter l'ecclesia des vivants pour être inhumé (fig. 3). 


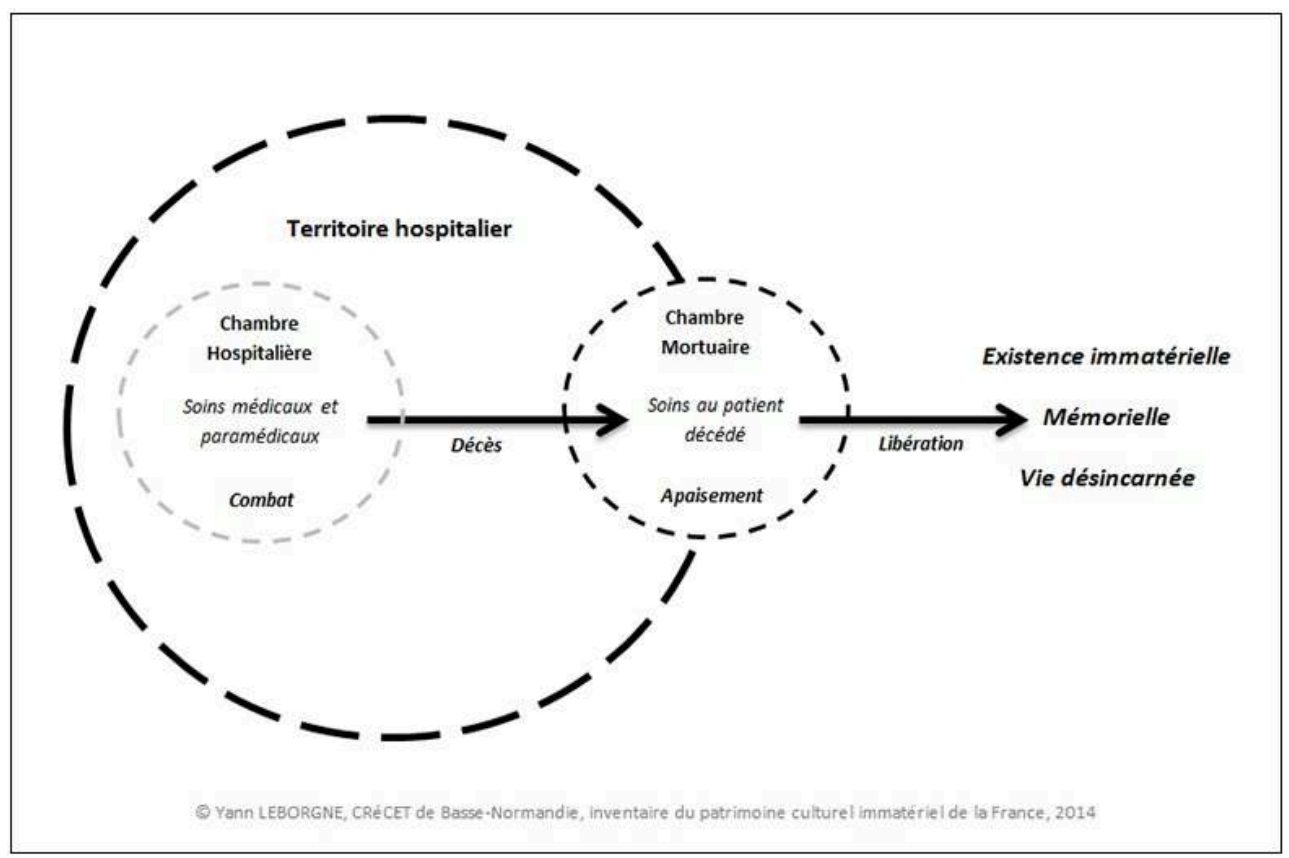

La chambre mortuaire, un seuil du territoire hospitalier

Dess. Leborgne, Yann, 2014. EPCC La Fabrique de patrimoines en Normandie. C CRéCET de BasseNormandie.

\section{Conclusion}

Les témoignages des hospitaliers rencontrés dans le cadre de cette étude révèlent que ce qui « fait patrimoine culturel immatériel » c'est d'abord une capacité à sauvegarder l'humanité de l'être là où celle-ci pourrait se voir dégradée par la maladie et la douleur. À cet égard, les pratiques à l'œuvre dans la chambre mortuaire du CHU de Rouen dévoilent combien un hôpital peut être le siège de résiliences ritualisées, y compris lorsque survient le décès d'un patient dans un service de soins. Il est remarquable qu'une telle approche investiguant le sens des métiers hospitaliers vienne au jour à l'heure où ces établissements, connaissant de profondes mutations en s'adaptant à la société globale, s'interrogent sur leur avenir et aspirent à se libérer d'une souffrance collective à laquelle ils sont en proie.

Ce travail illustre comment un inventaire du patrimoine culturel immatériel peut être mobilisé, en réponse à des enjeux sociaux et territoriaux sensibles. Il s'agit ici d'accompagner des communautés dans une re-connaissance d'elles-mêmes tandis que des mutations s'opèrent et bouleversent les anciens repères. L'investigation scientifique se conjugue alors avec une action culturelle et sociale. Pour reprendre une analogie médicale, cette démarche pourrait être apparentée à un "soin » adressé à un collectif qui éprouve une vive inquiétude existentielle. À l'heure où le système hospitalier public se restructure pour assurer sa pérennité, le processus patrimonial doit y restaurer un sentiment d'identité participant d'un mieux-être ensemble. En cela, cette opération est partie prenante d'une résilience.

Cette quête de patrimoine culturel immatériel n'est pas égocentrée et elle ne se confond pas avec une revendication identitaire. Bien qu'elle ait trait à une "quête de 
soi ", elle émane d'abord de communautés qui cherchent à se connaître pour mieux s'ouvrir et renaître au monde. Dans la mesure où elle procède d'une sauvegarde de l'humanité des soins comme de l'accueil de tous, elle réinterroge aussi la qualité de la prise en charge pour des patients qui, eux-mêmes, sont à l'hôpital en quête d'une délivrance. Ainsi, cette opération est-elle tournée vers l'autre: l'autre intérieur (on questionne le sens inconscient des actes soignants), l'autre extérieur (l'autre avec lequel on travaille), mais aussi l'inconnu qui est soigné parce qu'on lui reconnait avant tout son humanité.

Dans la mise en œuvre de cet inventaire, le travail de recherche scientifique occupe naturellement une place de premier ordre. Souvenons-nous qu' « inventaire » vient du latin inventarium (de invenire) qui signifie "trouver». Néanmoins le chercheur doit accepter de ne plus avoir le monopole de la science car, auprès de la communauté, il ne peut être celui qui sait. Il est d'abord un tiers révélateur. Ses techniques d'enquête diffusent l'investigation à l'intérieur d'un collectif qui, bien que s'interrogeant sur ce qu'il est, détient lui-même inconsciemment ce savoir. C'est à ce titre que les résultats de l'inventaire, bien qu'ils soient validés par un comité scientifique composé d'experts, ne peuvent exister sans l'aval et la relecture de ses contributeurs «non-experts». Ils sont le fruit d'une collaboration.

Cette démarche ne saurait être qu'un exercice réflexif. Reposant sur le vécu, elle ne peut atteindre son but auprès d'un large public qu'en parvenant à associer les dimensions émotionnelle et conceptuelle. Ceci implique de diversifier les registres de valorisation pour toucher le plus grand nombre et accroître l'accessibilité des découvertes. C'est en ce sens que la création artistique est désormais mobilisée dans le cadre des restitutions de cet inventaire hospitalier. La représentation d'une compagnie théâtrale à partir des témoignages recueillis ou encore la perspective prochaine d'une bande-dessinée et d'un travail photographique inspirés du contenu de la fiche d'inventaire nous rappellent que le patrimoine culturel immatériel n'est pas une construction intellectuelle réservée à un cercle étroit d'initiés. Le « $\mathrm{PCI}$ » s'adresse à tous car il est également un patrimoine du cœur.

\section{NOTES}

1. - Le texte intégral de la convention de 2003 pour la sauvegarde du patrimoine culturel immatériel est disponible sur le site internet de l'Unesco à l'adresse suivante: http:// www.unesco.org/culture/ich/fr/convention [consulté le 25/11/2016].

2. - On s'appuie ici sur la définition de la résilience développée par Christina ASCHAN-LEYGONIE. «Vers une analyse de la résilience des systèmes spatiaux ». L'espace géographique, 2000, n 29-1, p. 63-77.

3. - La loi sur la tarification à l'activité (dite «T2A») a transformé depuis le milieu des années 2000 le mode de financement des hôpitaux en les rémunérant sur la base des actes effectués sur les patients.

4. - Constat exprimé par les professionnels hospitaliers au cours des réunions préparatoires au projet patrimonial. 
5. - Ce travail se révèle comme un prolongement d'une approche plus spécifiquement mémorielle qui avait été engagée par Anne Monjaret et Marie-Christine Pouchelle [1999] dans trois hôpitaux parisiens (Boucicaut, Laennec et Broussais). Lire à ce propos MONJARET, Anne. «Fermeture et transfert de trois hôpitaux parisiens. L'ethnologue, accompagnateur social ». Ethnologie française. "Terrains minés ", 2001, nº1, p. 103-115.

6. - À travers notamment la notion de territorialité, au sens que lui accorde le géographe Claude Raffestin. La territorialité regroupe l'ensemble des manifestations par lesquelles les hommes expriment leur pouvoir sur un territoire en vue d'atteindre la plus grande autonomie possible. Lire à ce propos RAFFESTIN, Claude. "Repères pour une théorie de la territorialité humaine ". Groupe réseaux, 1987, $\mathrm{n}^{\circ}$ 7, p. 2-22.

7. - Définition énoncée oralement par ce médecin à l'occasion d'échanges préliminaires à l'inventaire.

8. - Voir BLOCH, Marc. Les Rois thaumaturges, étude sur le caractère surnaturel attribué à la puissance royale, particulièrement en France et en Angleterre [1924]. Paris : Gallimard, 1983.

9. - Des médecins et chirurgiens attachés à leur autorité comme à l'apparat d'un pouvoir de guérir.

10. - Nous reprenons ici la métaphore guerrière employée par Marie-Christine Pouchelle, dans Ead.. L'Hôpital ou le théâtre des opérations. Paris : Seli Arslan, 2008.

11. - Les lois de 2002 relatives à la qualité du système de santé et au droit des malades replacent d'ailleurs le patient au centre du dispositif et lui reconnaissent des droits fondamentaux.

12. - L'appel à témoignages a été diffusé dans les canaux d'information des CHU par leurs délégations culturelles.

13. - La fiche d'inventaire est consultable sur le site internet du ministère de la Culture : http:// www.culturecommunication.gouv.fr/Politiques-ministerielles/Patrimoine-culturel-immateriel/ Inventaire-en-France/Inventaire/Fiches-de-l-inventaire-du-patrimoine-culturel-immateriel.

14. - Précision soulignée par l'un des agents de la chambre mortuaire du CHU de Rouen.

15. - Voir témoignage $n^{\circ} 2$.

\section{RÉSUMÉS}

À l'heure où les hôpitaux universitaires connaissent d'importantes mutations économiques et organisationnelles, le CHU de Rouen s'est engagé dans un inventaire de son patrimoine culturel immatériel. Initié par la délégation à la culture de l'établissement, sous les conseils de la DRAC de Haute-Normandie, ce travail n'a pas été pensé comme une simple mesure de sauvegarde et de valorisation d'éléments constitutifs de l'identité d'une communauté hospitalière. Reposant sur la participation volontaire d'un grand nombre d'agents, il répondait à une quête existentielle autour des significations de leurs pratiques quotidiennes. Bien que l'entreprise s'inscrive dans la longue durée compte tenu de ses ambitions et du gigantisme du territoire concerné, cet article revient sur cette démarche qui souligne le caractère opératoire du patrimoine culturel immatériel. Appréhendé en tant que moyen d'introspection et de reconnaissance, son inventaire s'est trouvé investi par une communauté hospitalière afin de l'aider à surmonter sa souffrance au moment où des changements la conduisent à s'interroger sur ses fondements et ses valeurs. Le patrimoine culturel immatériel s'est ainsi dévoilé comme un soin intervenant dans un processus de résilience. 
At a time when university hospitals are experiencing significant economic and organisational changes, the $\mathrm{CHU}$ of Rouen has committed itself to carrying out an inventory of its intangible cultural heritage. The project was initiated by the hospital's cultural delegation with the support of the regional cultural services (DRAC Normandie). This project was not considered merely as a measure of heritage safeguarding, nor as the recognition of the constituent parts of the hospital community's identity. Based on the voluntary participation of many members of the hospital staff, it sought to answer the existential questions that surround the meaning of their everyday life practices. Though this undertaking was established over the long-term, according to its ambition and the vast scale of the territory concerned, this article looks back on an approach that underlines the operational aspects of an inventory of the intangible cultural heritage. Treated as a form of introspection and recognition, the inventory became invested by the hospital community in order to help it overcome the pain, when changes lead to questions about inner principles and values. The intangible cultural heritage emerged as a cure taking part in a process of resilience.

\section{INDEX}

Keywords : intangible cultural heritage, resilience, hospital, territoriality, ritual practice, Rouen, Angers

Mots-clés : patrimoine culturel immatériel, résilience, hôpital, territorialité, pratique rituelle, Rouen, Angers

\section{AUTEUR}

\section{YANN LEBORGNE}

Chercheur chargé de mission pour l'inventaire et la sauvegarde du patrimoine culturel immatériel, Ethnopôle « La Fabrique de patrimoines en Normandie » yann.leborgne@lafabriquedepatrimoines.fr 